\title{
Insights into the relation between crystal structure and deuterium desorption characteristics of Pd-Au-D alloys
}

\author{
D.E. Nanu ${ }^{\text {a) }}$ \\ W.J. Legerstee ${ }^{\text {b) }}$ \\ S.W.H. Eijt ${ }^{\text {) }}$ \\ W.G. Haije \\ J.F. Vente \\ M.G. Tucker ${ }^{\text {c) }}$ \\ A.J. Böttger ${ }^{\text {a) }}$
}

a) Delft University of Technology, Faculty of Mechanical, Maritime and Materials Engineering

b) Delft University of Technology, Faculty of Applied Sciences

c) ISIS Facility 


\title{
Insights into the relation between crystal structure and deuterium desorption characteristics of $\mathrm{Pd}-\mathrm{Au}-\mathrm{D}$ alloys
}

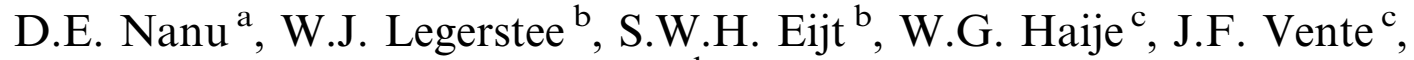 \\ M.G. Tucker ${ }^{\mathrm{d}}$, A.J. Böttger ${ }^{\mathrm{a}, *}$ \\ a Department of Materials Science and Engineering, Faculty of Mechanical, Maritime and Materials Engineering, Delft University of Technology, \\ Mekelweg 2, 2628 CD Delft, The Netherlands \\ ${ }^{\mathrm{b}}$ Department of Radiation, Radionuclides and Reactors, Faculty of Applied Sciences, Delft University of Technology, Delft, The Netherlands \\ ${ }^{\mathrm{c}}$ Energy research Centre of the Netherlands, Petten, The Netherlands \\ ${ }^{\mathrm{d}}$ ISIS Facility, Rutherford Appleton Laboratory, Chilton, Didcot, Oxfordshire, UK
}

Received 16 July 2008; received in revised form 13 August 2008; accepted 13 August 2008

Available online 1 October 2008

\begin{abstract}
Neutron diffraction and thermal desorption spectroscopy are used to determine the details in the crystallographic structure and the deuterium desorption characteristics of deuterated $\mathrm{Pd}-\mathrm{Au}$ alloys. Compositions under investigation include alloys corresponding to both subcritical and supercritical conditions with respect to the $\alpha-\beta$ phase transitions. The effect of the Au-content on deuterium desorption properties is discussed in relation to the degree of deuterium loading and the phase diagram. Furthermore, the distribution of deuterium over the octahedral and tetrahedral interstitial sites is determined. The tetrahedral site occupancy increases with increasing Au content and is significant for both supercritical and subcritical phases.

(C) 2008 Acta Materialia Inc. Published by Elsevier Ltd. All rights reserved.
\end{abstract}

Keywords: Transition metals; Interstitials; Hydrogen desorption; Neutron diffraction; Site occupancy

\section{Introduction}

One of the main technical challenges associated with the production of high-purity hydrogen at reduced costs is the development of highly selective hydrogen membranes. These membranes should withstand the demanding industrial conditions of high temperatures and pressures in multicomponent gaseous environments. Supported dense Pd-based membranes with a thickness of several micrometres are the most suitable options for this purpose [1]. However, the lifetime of pure Pd membranes in thermal cycles from operational conditions to room temperature is limited. Failure often occurs owing to deformation and fracture caused by the large specific volume change during

\footnotetext{
* Corresponding author. Tel.: +31 15278 2243; fax: +31 152786730.

E-mail address: A.J.Bottger@tudelft.nl (A.J. Böttger).
}

the hydride formation and decomposition processes. Several experimental and theoretical studies suggest that a longer operation lifetime can be achieved through the addition of specific alloying elements [1,2]. A lifetime in the order of years is essential for an economically viable process. Beneficial effects of alloying consist of: a reduction of the critical temperature for the transition from the $\alpha$ metal solid solution to the $\beta$-hydride phase, a smaller unit cell volume difference between the two phases, improved mechanical properties, and an enhanced resistance to surface poisoning, e.g., by sulfur compounds [1,2]. Furthermore, higher hydrogen solubility and diffusivity for many $\mathrm{Pd}$ alloys (e.g., alloys with $\mathrm{Ag}, \mathrm{Au}$ or $\mathrm{Cu}$ ) result in higher hydrogen permeability than in $\mathrm{Pd}$ itself [2]. The hydrogen solubility and the diffusion mechanism of hydrogen through these membranes are determined by the nature of the specific alloying elements, their concentration and distribution in the metal lattice, as well as by the (local) 
interaction of the hydrogen atoms with the alloying elements. Structural information of the phases formed during hydrogen absorption/desorption in Pd alloys is therefore crucial for understanding the effect of alloying on macroscopic hydrogen permeation behaviour. This formed the main motivation for investigating the effect of the crystal structure of hydrogenated (deuterated) Pd alloys and its relation with the desorption characteristics. $\mathrm{Pd}-\mathrm{Au}$ alloys were chosen as a model system for this work, because they fulfil the requirements for promising membrane materials, and they are expected to show characteristic phenomena such as hydrogen-induced (short- and long-range) ordering transformations [3], interesting also from a fundamental point of view.

Pd and many of its alloys (with small content of alloying elements) crystallize in the face centred cubic (fcc) structure [4]. Two distinct types of interstitial sites available to hydrogen atom occupancy can be distinguished, namely the octahedral $(\mathrm{O})$ and tetrahedral $(\mathrm{T})$ positions. For the palladium-hydrogen system, studied for more than a century, it is commonly accepted that hydrogen atoms occupy exclusively the octahedral interstitial sites. This has been confirmed by early neutron diffraction (ND) studies on palladium-deuterium samples [5] and accepted by extension for the case of hydrogen atoms. A number of publications reporting ND studies on $\mathrm{Pd}-\mathrm{H}(\mathrm{D})$ powders [5-8] or single crystals $[9,10]$, and quasielastic neutron scattering studies of the hydrogen dynamics in palladium $[11,12]$ support this conclusion. However, most of these structural studies have considered in their data analysis only the occupation of either the octahedral or the tetrahedral sites solely, and have shown that, within the limits of these two options, the O-site-only occupancy is most consistent with the experimental data. This clearly leaves open the occurrence of simultaneous occupation of $\mathrm{O}$ and $\mathrm{T}$ sites, which could possibly provide a better description of the ND data, especially at higher $H(D)$ contents. Nevertheless, the hypothesis of octahedral site occupancy was further generalized for hydrogen in fcc Pd alloys. To the best of the authors' knowledge, however, there is no experimental evidence confirming this hypothesis for all relevant cases. In particular, for $\mathrm{Pd}-\mathrm{Au}$ alloys, the limited amount of available ND data are from room temperature experiments and were interpreted in terms of O-site-only occupation in the $\alpha$ phase [13]. To what extent this is valid for $\mathrm{Pd}-\mathrm{Au}$ alloys with different Au contents and higher H(D) concentrations is therefore still unknown.

Theoretical studies on hydrogen in Pd and Pd alloys, in contrast, suggest that, depending on the alloying element and its concentration, the occupation of tetrahedral sites may occur. Moreover, it has also been suggested that $\mathrm{T}$ sites could act as transition states in the process of hydrogen diffusion. For hydrogen in pure $\mathrm{Pd}$, first principles calculations have shown that, while occupation of $\mathrm{T}$ sites by hydrogen atoms is less favourable than occupation of $\mathrm{O}$ sites, because the potential energy of hydrogen at the $\mathrm{T}$ site is slightly higher than at the $\mathrm{O}$ site, hydrogen diffusion between $\mathrm{O}$ sites necessarily occurs via passage of $\mathrm{T}$ sites. Furthermore, significant self-trapping of hydrogen at the T-site position can be induced, depending on the magnitude of both the local relaxation of $\mathrm{Pd}$ atoms around the $\mathrm{T}$ site and the vibrational energy of the interstitial species (H, D, T) [14-16]. Similar conclusions were drawn for $\mathrm{H}$ in Pd alloys. First principle calculations show that, in these cases, the local atomic arrangement of metal atoms in the nearest neighbourhood of the interstitial site is the dominant factor for the site occupancy and stability [17-20].

Experimental evidence supporting the simultaneous occupation of $\mathrm{O}$ and $\mathrm{T}$ sites is scarce and mostly controversial. Quasielastic neutron scattering measurements on $\beta$-Pd hydrides could be best described with a mixed occupation of $\mathrm{O}$ and $\mathrm{T}$ sites [21]. The proposed mechanism for hydrogen diffusion through palladium by hopping of $\mathrm{H}$ from $\mathrm{O}$ site to $\mathrm{O}$ site via intermediate $\mathrm{T}$ sites [21], rather than by direct $\mathrm{O}-\mathrm{O}$ or $\mathrm{T}-\mathrm{T}$ jumps as previously considered $[11,12]$, sparked an extensive scientific discussion. An important step forward was made in recent in situ ND investigations on the $\mathrm{Pd}-\mathrm{D}$ system in the supercritical region [22], presenting evidence that part of the deuterium atoms indeed occupy tetrahedral positions. In analysing their data, these authors considered a mixed octahedral and tetrahedral occupation, confirming conclusions of diffusion experiments [21] and first principles studies [14-16]. An important question is whether and to what extent T-site occupancy occurs in fcc Pd-based alloys, and whether it is limited to the supercritical region only, as inferred in Ref. [22] for the case of $\mathrm{Pd}$.

The present study is designed to provide an answer to these questions. ND experiments were performed to investigate the crystal structure of deuterated $\mathrm{Pd}-\mathrm{Au}$ alloys. The samples contained up to 25 at.\% $\mathrm{Au}$ and were loaded with different amounts of deuterium at room temperature. Sample compositions in both subcritical and supercritical regions were selected on the basis of the room temperature ternary phase diagram (Fig. 1). Deuterium was used instead of hydrogen to avoid a high background due to the incoherent scattering of hydrogen. The $\mathrm{Pd}-\mathrm{Au}-\mathrm{D}$ system is very well suited for ND studies, as the constituent elements have sufficiently different cross sections for coherent neutron scattering (i.e., $b_{\mathrm{Pd}}=0.591$ barn, $b_{\mathrm{Au}}=0.763$ barn, $b_{\mathrm{D}}=0.6674$ barn).

The crystallographic properties determined by ND experiments are complemented by the hydrogen desorption characteristics obtained by thermal desorption spectroscopy (TDS) on the same materials. The combined information obtained from TDS and ND provides a clear insight into the relation between the effect of alloying on macroscopic properties and on the microscopic atomic structure, as discussed in this paper.

\section{Experimental}

Pd foils (99.95\%, Goodfellow Metals) and foils of Pd$\mathrm{Au}$ alloys containing 10, 20 and 25 at.\% $\mathrm{Au}$ (Philips 


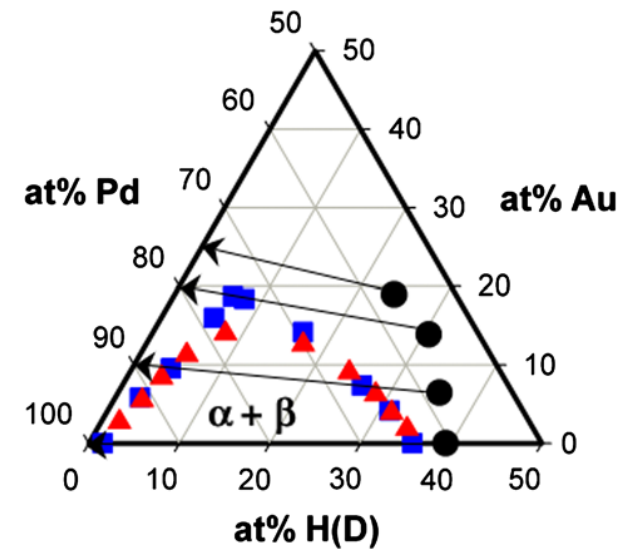

Fig. 1. Ternary phase diagram of $\mathrm{Pd}-\mathrm{Au}-\mathrm{H}$ system at room temperature and $0.1 \mathrm{MPa}$. Compositions are expressed as total at. $\%$ of elements in the ternary system $\operatorname{Pd}_{(100-x-y)} \mathrm{Au}_{x} \mathrm{H}_{y}$. The squares [30] and triangles [31] represent experimental phase boundaries based on literature data, using the correlation between $\mathrm{H}$ content and the associated volume expansion from Ref. [33]. The solid circles represent the initial compositions of $\operatorname{Pd}_{(100-x-y)} \mathrm{Au}_{x} \mathrm{D}_{y}$ samples investigated by TDS and for which the desorption profiles are given in Fig. 3. The change in composition during TDS is indicated by arrows.

Research Laboratory, Eindhoven) were used. The thickness of the foils was either $\sim 50 \mu \mathrm{m}$ or $\sim 100 \mu \mathrm{m}$. The composition of the $\mathrm{Pd}-\mathrm{Au}$ alloys was confirmed (to within $10 \%$ accuracy) by electron probe microanalysis and is given in Table 1 . The foils were chemically etched with a solution of $2: 2: 1 \quad \mathrm{H}_{2} \mathrm{SO}_{4} / \mathrm{HNO}_{3} / \mathrm{H}_{2} \mathrm{O}$ to remove any oxides from the surface, rinsed in acetone, and then annealed at $1073 \mathrm{~K}$ for $2 \mathrm{~h}$ under vacuum. Deuterium gas $(99.7 \%$, Linde AG) was used for loading the samples.

\subsection{Thermal desorption measurements}

TDS measurements were performed on samples of $\operatorname{Pd}_{(100-x)} \mathrm{Au}_{x}$ alloys for $x$ in the range $0-25$ at.\% loaded at room temperature using a deuterium pressures of 0.6 or $1.0 \mathrm{MPa}$. A homebuilt thermal desorption set-up, HYDRA, coupled with a quadrupole mass spectrometry (QMS) device for sensitive deuterium detection [23] was used for this purpose. In order to achieve a highly active state of the samples prior to deuterium loading, the reaction chamber was completely outgassed at $575 \mathrm{~K}$ and $\sim 2 \times 10^{-3} \mathrm{~Pa}$, then exposed to $0.4 \mathrm{MPa}$ deuterium gas for $15 \mathrm{~min}$, and subsequently cooled down to room temperature and de-deuterated by evacuating and reheating to $575 \mathrm{~K}$. This activation procedure was repeated twice and followed by complete degassing for $30 \mathrm{~min}$ at $650 \mathrm{~K}$ in vacuum. The samples were subsequently loaded with deuterium at $303 \mathrm{~K}$ and a constant pressure of 0.6 or $1.0 \mathrm{MPa}$ $\mathrm{D}_{2}$ for $4 \mathrm{~h}$. This time was found to be sufficient to achieve equilibrium. After deuterium loading, the reaction chamber was cooled quickly to $150 \mathrm{~K}$, degassed and, subsequently, a thermal desorption spectrum was recorded. A linear temperature ramp with a rate between 0.4 and $0.7 \mathrm{~K} \mathrm{~min}^{-1}$ was used, depending on the specific sample. Calibration of the QMS deuterium signal was performed after each desorption run by exposure to a well-determined quantity of deuterium released from a reference container with a calibrated volume. Deuterium fractions were extracted from the integrated thermal desorption profiles, and the weight of the samples was determined before the TDS measurements.

\subsection{ND measurements}

Samples containing $\sim 3 \mathrm{~g}$ Pd alloy were prepared for ND measurements. The samples consist of alloy foils cut as discs $5 \mathrm{~mm}$ in diameter and arranged in a cylindrical stack $\sim 15 \mathrm{~mm}$ high. The discs were chemically etched, annealed, activated and charged by similar procedures to those used for the thermal desorption measurements.

After deuterium loading for $4 \mathrm{~h}$ at a pressure of 0.6 or 1.0 $\mathrm{MPa} \mathrm{D}_{2}$ at $303 \mathrm{~K}$, the reaction chamber was quickly

Table 1

Deuterium-free $\operatorname{Pd}_{(100-x)} \mathrm{Au}_{x}$ samples: composition, lattice parameters and Rietveld fit parameters

\begin{tabular}{|c|c|c|c|c|c|c|c|c|c|}
\hline Alloy & $\begin{array}{l}x^{*} \\
\text { (at.\%) }\end{array}$ & $\begin{array}{l}a_{\text {lit }} \times 10^{10} \\
(\mathrm{~m})\end{array}$ & $\begin{array}{l}a \times 10^{10} \\
(\mathrm{~m})\end{array}$ & $\begin{array}{l}B_{\mathrm{M}} \times 10^{20} \\
\left(\mathrm{~m}^{2}\right)\end{array}$ & Texture index & No. of parameters & $w R p$ & $\chi^{2}$ & Refinement strategy \\
\hline $\mathrm{Pd}_{90} \mathrm{Au}_{10}$ & 9.9 & $3.911(1)$ & $3.91026(2)$ & $\begin{array}{l}0.47 \\
0.47 \\
0.52(1)\end{array}$ & $\begin{array}{l}1.33 \\
1.00 \\
1.30\end{array}$ & $\begin{array}{l}71 \\
65 \\
72\end{array}$ & $\begin{array}{l}0.0705 \\
0.0776 \\
0.0701\end{array}$ & $\begin{array}{l}6.77 \\
8.19 \\
6.70\end{array}$ & $\begin{array}{l}\text { (a) } \\
\text { (b) } \\
\text { (c) }\end{array}$ \\
\hline $\mathrm{Pd}_{80} \mathrm{Au}_{20}$ & 19.8 & $3.930(1)$ & $3.92612(3)$ & $\begin{array}{l}0.48 \\
0.48 \\
0.60(1)\end{array}$ & $\begin{array}{l}1.07 \\
1.00 \\
1.05\end{array}$ & $\begin{array}{l}71 \\
65 \\
72\end{array}$ & $\begin{array}{l}0.1133 \\
0.1247 \\
0.1121\end{array}$ & $\begin{array}{l}5.44 \\
6.59 \\
5.33\end{array}$ & $\begin{array}{l}\text { (a) } \\
\text { (b) } \\
\text { (c) }\end{array}$ \\
\hline $\mathrm{Pd}_{75} \mathrm{Au}_{25}$ & 25.0 & $3.9400^{* *}$ & $3.93587(2)$ & $\begin{array}{l}0.49 \\
0.49 \\
0.56(1)\end{array}$ & $\begin{array}{l}1.05 \\
1.00 \\
1.04\end{array}$ & $\begin{array}{l}71 \\
65 \\
72\end{array}$ & $\begin{array}{l}0.0670 \\
0.0717 \\
0.0675\end{array}$ & $\begin{array}{l}4.20 \\
4.80 \\
4.25\end{array}$ & $\begin{array}{l}\text { (a) } \\
\text { (b) } \\
\text { (c) }\end{array}$ \\
\hline
\end{tabular}

The three sets of parameters given for each sample correspond to (a) refinements using fixed average values for the temperature factors and including texture refinement, (b) refinements using fixed average values for the temperature factors without texture refinement, and (c) refinements including temperature factors and texture refinement. The numbers in parentheses are the estimated standard deviation in the last quoted digit, as given in GSAS for the refined parameters.

* $10 \%$ accuracy.

** Estimated based on linear regression $a=\mathrm{f}(\mathrm{at} . \% \mathrm{Au})$ of data from Ref. [30]. 
cooled to $150 \mathrm{~K}$ and degassed. The samples were subsequently removed and immediately rinsed with an aqueous mixture of $0.1 \mathrm{M}$ thiourea and $0.1 \mathrm{M} \mathrm{NaOH}$ solutions, and then immersed in a xylene solution containing $0.1 \mathrm{~g}$ polyethylene and $1 \mathrm{~g}$ sulfur to poison the surface. The last step was designed to block the surface of the specimens in order to reduce dehydrogenation in the time interval between deuterium loading and the ND studies to a sufficiently low level. Subsequently, the specimens where placed in a ceramic container and sealed in a stainless steel can under deuterium gas at the same pressure as used for deuterium loading.

ND data were obtained using the general materials (GEM) diffractometer at the ISIS pulsed neutron source located at the Rutherford Appleton Laboratory UK [24]. For the ND measurements, the specimens were transferred from the ceramic container into a vanadium sample holder and sealed in air at atmospheric pressure just before the start of each experiment. All samples were measured for $\sim 1 \mathrm{~h}$ at room temperature, diffraction patterns being recorded simultaneously by five detector banks positioned at $2 \theta$ of $154.4,91.3,63.9,35$ and $18^{\circ}$, respectively. To obtain insight into the stability of the alloys, the samples of $\mathrm{Pd}_{90} \mathrm{Au}_{10}$ and $\mathrm{Pd}_{75} \mathrm{Au}_{25}$ loaded at $0.6 \mathrm{MPa}$ were measured for over $8 \mathrm{~h}$ in 1 -h data collections. No significant differences between the first and the final patterns were observed. This indicates that no deuterium losses occurred during the diffraction experiments.

\subsection{ND data handling}

The ND patterns were analysed by the Rietveld method using the General Structure Analysis System (GSAS) software package [25]. The patterns from the different detector banks (each giving a different and partly overlapping range of accessible $d$-spacing from 0.03 to $0.5 \mathrm{~nm}$ ) were fitted simultaneously with the lattice parameters, deuterium site occupancies, temperature factors and texture parameters as refinable structural parameters. The effect of texture on the structure factors was corrected using 8th-order spherical harmonics coefficients for samples with cylindrical symmetry, as implemented in GSAS. Random orientation (texture index $=1$ ) was assumed as an initial guess for the texture parameters. The background of each histogram was described with a shifted Chebyshev function with eight parameters. The second TOF profile function from GSAS was used to describe the diffraction profiles, two profile parameters (sig-1 and gam-1) being refined for each histogram. In addition, one absorption correction related parameter and one histogram scaling parameter were used for each histogram. Bank $6\left(2 \theta=154.4^{\circ}\right)$ was selected as the reference bank to which the instrumental parameters (DIFA and DIFC) describing the characteristic length of the flight paths of the other banks were normalized. Several refinement procedures were considered to ensure that the data analysis was objective and unbiased, and that the unavoidable correlations between the interstitial site occu- pancies, temperature factors and texture parameters were handled properly. No systematic correlations larger than 0.5 were present between the relevant structural parameters. Details on the refinements and their results are given in Section 3.3.

\section{Results and discussion}

\subsection{Deuterium loading of $P d_{(100-x)} A u_{x}$ alloys (foils)}

The deuterium content in the metal foils after loading at 0.6 and 1.0 MPa was determined two to four times, using fresh samples for each of the $\operatorname{Pd}_{(100-x)} \mathrm{Au}_{x}$ compositions. Fig. 2 shows the measured deuterium content as a function of the $\mathrm{Au}$ content in $\operatorname{Pd}_{(100-x)} \mathrm{Au}_{x}$. The error bars are representative for the spread in measured fractions for a specific condition. The spread in data can be related to the experimental accuracy of the desorption set-up in the determination of deuterium fraction and to possible small variations in the microstructure and surface properties of the foils.

The fraction of incorporated deuterium gradually decreases as a function of $\mathrm{Au}$ fraction in agreement with the expected trend, i.e., isobaric hydrogen solubilities decrease with increasing fraction of alloying element [26]. Also the results from previous studies on electrochemically loaded $\mathrm{Pd}-\mathrm{Au}$ electrodes [27], represented for comparison by the line in Fig. 2, show the same trend. This systematic decrease in hydrogen absorption capacity is probably related to the fact that, for higher loading fractions, deuterium atoms progressively need to occupy less favourable binding sites. Consequently, this limits the attainable maximum absorbed deuterium fractions in $\mathrm{Pd}-\mathrm{Au}$ alloys relative to palladium at a given deuterium loading pressure and temperature.

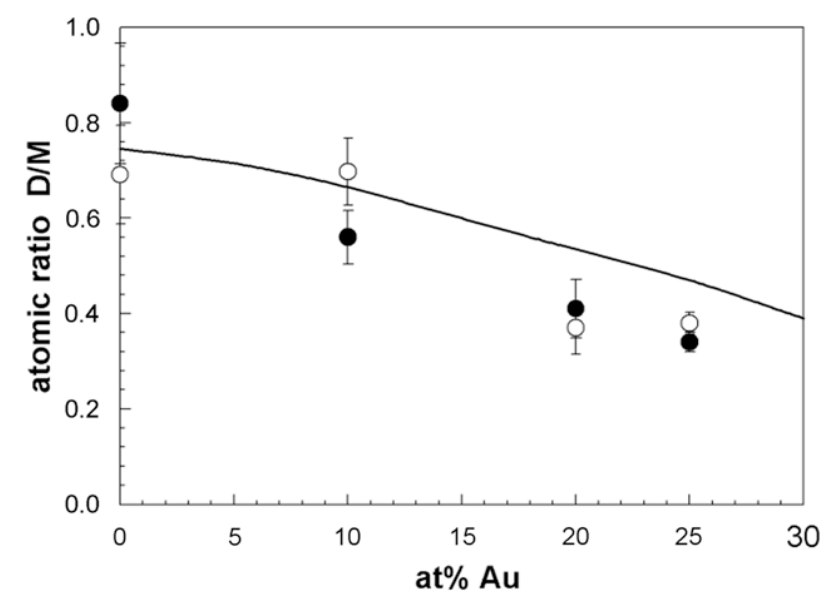

Fig. 2. Fraction of deuterium (D atoms/metal atoms) loaded in $\operatorname{Pd}_{(100-x)} \mathrm{Au}_{x}$ foils by exposure to $0.6 \mathrm{MPa}$ (open symbols) and $1.0 \mathrm{MPa}$ (solid symbols) deuterium gas at room temperature for $4 \mathrm{~h}$, as determined from subsequent thermal desorption. Line: data from Ref. [27] corresponding to electrochemical loading. 


\subsection{Deuterium TDS on $P d_{(100-x)} A u_{x}$ foils}

The position of desorption peaks and the shape of the TDS profiles depend on the heating rate and can provide information on the local binding energy of hydrogen or deuterium in metals [28]. Typical deuterium thermal desorption spectra of $\operatorname{Pd}_{(100-x)} \mathrm{Au}_{x}$ alloys loaded at 1.0 $\mathrm{MPa}$ are shown in Fig. 3. The starting compositions of these samples correspond to single-phase systems with a deuterium content of $0.65,0.55,0.44$ and $0.32 \mathrm{D} /$ metal atomic ratio for $\operatorname{Pd}_{(100-x)} \mathrm{Au}_{x}$ alloys with $x$ equal to 0 , 10,20 and 25 at. $\%$, respectively. These compositions are represented by black solid circles in the ternary phase diagram (Fig. 1).

The TDS profile obtained for the Pd reference sample $(x=0)$ consists of a relatively broad low temperature shoulder, between 300 and $350 \mathrm{~K}$, followed by a much sharper desorption peak. This shape is characteristic of desorption from Pd samples loaded beyond the $\alpha-\beta$ coexistence region into the pure $\beta$-hydride phase, i.e., for hydrogen fractions beyond $\sim 0.6$ [29]. This is indeed the case for the reference sample (Fig. 1). The low temperature shoulder is related to deuterium release from a single-phase interstitial solid solution: here the $\beta$-Pd hydride. The relative broad nature of this shoulder is indicative for desorption from multiple trap sites with comparable binding

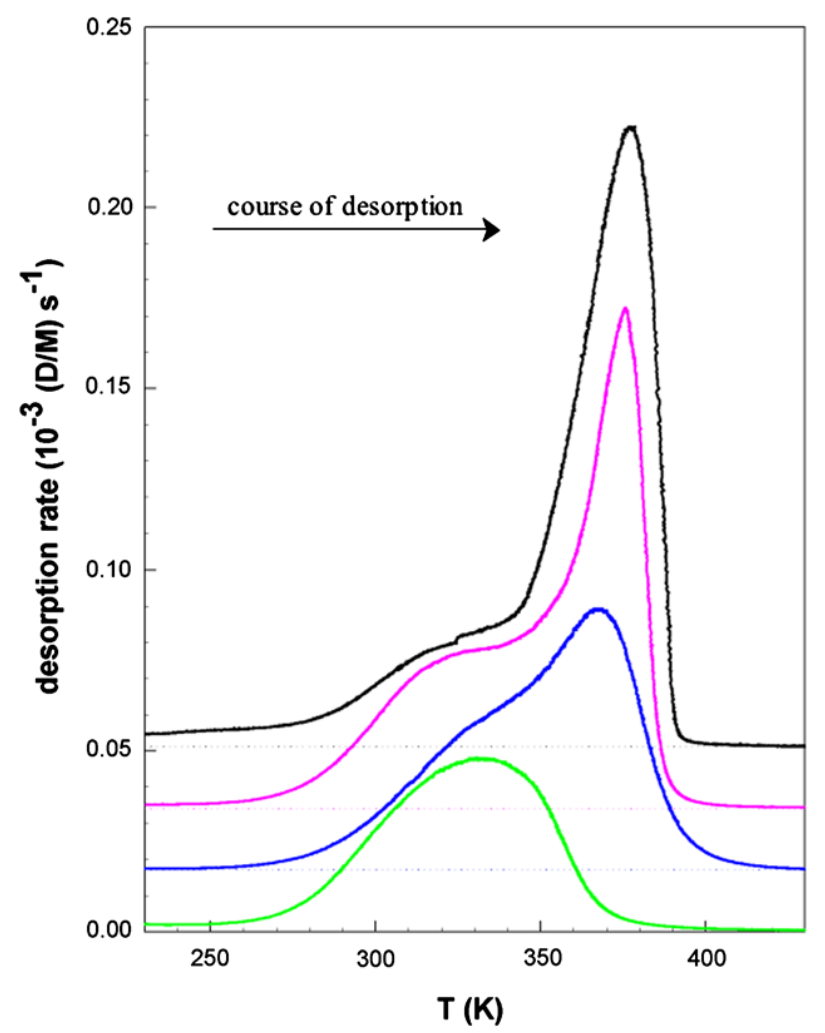

Fig. 3. Deuterium TDS profiles of $\operatorname{Pd}_{(100-x)} \mathrm{Au}_{x}$ samples charged by exposure to $1.0 \mathrm{MPa}$ deuterium at room temperature for $4 \mathrm{~h}$. From top to bottom, the profiles correspond to $x=0,10,20$ and 25 , respectively, corresponding to the compositions represented by solid circles in Fig. 1. energies [28]. The sharp TDS peak near $380 \mathrm{~K}$ is related to the release of deuterium from a system comprising two coexisting phases created by the hydride decomposition, i.e., desorption occurs via a $\beta \rightarrow \alpha$ phase transformation step. The corresponding desorption peak is quite narrow, suggesting desorption of hydrogen from a single-trap state, with the rate-determining step probably being the movement of the $\alpha-\beta$ interface. The higher desorption temperature peak is therefore most likely related to the onset of the hydride decomposition.

The shape of the TDS profiles from alloys with different Au content $(x=10,20$ and 25 at.\%) changes systematically, with a typical evolution as shown in Fig. 3. In particular, the profiles for the alloy composition $\mathrm{Pd}_{75} \mathrm{Au}_{25}$ are remarkably different. The desorption profiles from $\mathrm{Pd}_{90} \mathrm{Au}_{10}$ and $\mathrm{Pd}_{80} \mathrm{Au}_{20}$ are similar to those from $\mathrm{Pd}$ foils, indicating that these samples were also loaded beyond the $\alpha-\beta$ coexistence region. Indeed, the compositions of these samples at the beginning of the TDS measurement correspond to single $\beta$ hydride phases (see solid circles in Fig. 1, and the arrows indicate the changes in composition during desorption). Similarly to the case of pure Pd, hydrogen desorption from $\mathrm{Pd}_{90} \mathrm{Au}_{10}$ and $\mathrm{Pd}_{80} \mathrm{Au}_{20}$ alloys proceeds first with deuterium being released from the interstitial sites of the single-phase $\beta$-hydride (low temperature shoulder), followed by desorption from a system consisting of coexisting $\alpha$ and $\beta$ domains with deuterium release occurring accompanied by a $\beta \rightarrow \alpha$ phase transformation reaction (desorption peak at higher temperatures). With increasing Au fraction, the high temperature desorption peak becomes broader. This is probably the consequence of a decrease in hydrogen (deuterium) diffusivity with increasing $\mathrm{Au}$ fraction due to the existence of interstitial sites that could either be unfavourable for H(D) binding or provide too strong a binding for $\mathrm{H}(\mathrm{D})$ (trapping sites). First principles calculations $[17,18]$ support this argument, showing that the local alloy composition in the nearest (nn) and next-nearest (nnn) neighbourhood of an interstitial site is determining the local hydrogen binding energy, resulting in forbidden and trapping sites. For example, octahedral sites with local alloy configuration $(\mathrm{nn}, \mathrm{nnn})$ of $(0,7),(3,0)(3,3) \mathrm{Au}$ atoms bind hydrogen atoms more strongly than sites with a local composition consisting of $\mathrm{Pd}$ only, i.e., $(\mathrm{nn}, \mathrm{nnn})=(0,0)$, whereas sites with $(6,0)$ and $(5,2)$ Au atoms are unfavourable for $\mathrm{H}$ (forbidden occupations) [17].

The desorption profiles from $\mathrm{Pd}_{75} \mathrm{Au}_{25}$ foils consist of only one broad peak centred at low temperatures. The absence of the high temperature peak characteristic to deuterium release via the hydride decomposition reaction infers that in this case deuterium desorption takes place without passing through the two coexisting-phases system. This is consistent with the fact that the composition of this sample corresponds to a single supercritical phase (Fig. 1). The position of the desorption peak from $\operatorname{Pd}_{75} \mathrm{Au}_{25}$ appears to be in the neighbourhood of the single-phase $\beta$-hydride shoulder of $\mathrm{Pd}-\mathrm{D}$ spectra, its shape being 
characteristic for thermal desorption from a single-phase system, including a range of interstitial trap sites with close but increasing binding energies. This implies that desorption from the interstitials sites in the single supercriticalsolid solution phase and in the single subcritical $\beta$-hydride phase have very similar characteristics.

In the view of the above interpretation of the TDS profiles, the present study confirms that no hydride phase separation occurs during hydrogen (deuterium) absorption/ desorption in $\mathrm{Pd}_{75} \mathrm{Au}_{25}$ alloys at $303 \mathrm{~K}$. The hydride phase separation still does take place in $\mathrm{Pd}_{80} \mathrm{Au}_{20}$. This indicates that the critical composition for the disappearance of the $\alpha-\beta$ miscibility gap at room temperature is $\sim 21$ at. $\% \mathrm{Au}$ [30] rather than 17 at.\% $\mathrm{Au}[31]$. Thus, $\beta$-hydride phase formation occurs upon deuterium loading of $\mathrm{Pd}, \mathrm{Pd}_{90} \mathrm{Au}_{10}$, $\mathrm{Pd}_{80} \mathrm{Au}_{20}$, at $303 \mathrm{~K}\left(<T_{\mathrm{c}}\right)$, while the loading of $\mathrm{Pd}_{75} \mathrm{Au}_{25}$ under these experimental conditions leads to the formation of a supercritical solid solution phase $\left(T>T_{\mathrm{c}}\right)$.

\subsection{Deuterium site occupancy determined from ND measurements}

The following paragraphs discuss the ND patterns of both the pure alloys (D-free) and the deuterated alloys. The D-free samples are included because they are essential for the interpretation of the deuterated samples, as the dis- cussion would not be complete if the case of pure alloys were not described.

ND patterns obtained on the pure alloys show that they crystallize, as expected, in the single-phase fcc structure (space group $F m \overline{3} m$ ). With increasing Au content, the unit cell expands. The refined lattice parameters are in good agreement with the literature data [31], as given in Table 1. The same values of the refined lattice parameters are obtained independently by all refinement strategies considered. Three different refinement strategies were employed: (a) refinements whereby the metal temperature factors are fixed to the average values of the temperature factors of the constituent elements and all other parameters are refined with texture corrections; (b) the same but without refining the texture; and (c) refinements whereby the temperature factors are refined together with texture parameters and all other parameters. In the refinements with fixed metal temperature factors $\left(B_{\mathrm{M}}\right)$, their values were estimated as weighted averages of the temperature factors of pure Pd and pure Au at $298 \mathrm{~K}$. The values of the DebyeWaller factors of pure elemental crystals given as functions of temperature in Ref. [32] were used to estimate the temperature factors of pure metals at $298 \mathrm{~K}$.

The results of the different refinements considered for the case of pure alloys are summarized in Table 1. These data indicate that the fit quality does not significantly differ

Table 2

Deuterium-loaded $\mathrm{Pd}_{(100-x)} \mathrm{Au}_{x}$ samples: composition (D/M atomic ratio), lattice parameters and Rietveld fit parameters

\begin{tabular}{|c|c|c|c|c|c|c|c|c|c|c|c|c|}
\hline \multirow[t]{2}{*}{ Sample } & \multirow[t]{2}{*}{$\mathrm{D} / \mathrm{M}_{\mathrm{TDS}}$} & \multirow{2}{*}{$\begin{array}{l}a \times 10^{10} \\
(\mathrm{~m})\end{array}$} & \multirow{2}{*}{$\begin{array}{l}B_{\mathrm{M}} \times 10^{20} \\
\left(\mathrm{~m}^{2}\right)\end{array}$} & \multirow{2}{*}{$\begin{array}{l}B_{\mathrm{D}} \times 10^{20} \\
\left(\mathrm{~m}^{2}\right)\end{array}$} & \multicolumn{2}{|c|}{ D occupancy } & \multirow[t]{2}{*}{$\mathrm{D} / \mathrm{M}_{\mathrm{ND}}$} & \multirow{2}{*}{$\begin{array}{l}\text { Texture } \\
\text { index }\end{array}$} & \multirow{2}{*}{$\begin{array}{l}\text { No. of } \\
\text { parameters }\end{array}$} & \multirow[t]{2}{*}{$w R \mathrm{p}$} & \multirow[t]{2}{*}{$\chi^{2}$} & \multirow{2}{*}{$\begin{array}{l}\text { Refinement } \\
\text { strategy }\end{array}$} \\
\hline & & & & & $\mathrm{O}$ & $\mathrm{T}$ & & & & & & \\
\hline \multicolumn{13}{|c|}{ Loading pressure $=0.6 \mathrm{MPa}$} \\
\hline \multirow[t]{3}{*}{$\mathrm{Pd}_{90} \mathrm{Au}_{10}$} & $0.70 \pm 0.07$ & $4.01948(3)$ & 0.47 & 3.80 & $0.499(2)$ & $0.023(1)$ & 0.55 & 1.62 & 73 & 0.0512 & 2.51 & (a) \\
\hline & & & 0.47 & 3.80 & $0.473(1)$ & $0.011(1)$ & 0.50 & 1.00 & 67 & 0.0601 & 3.45 & (b) \\
\hline & & & $0.53(1)$ & $3.64(4)$ & $0.478(3)$ & $0.021(1)$ & 0.52 & 1.51 & 75 & 0.0510 & 2.49 & (c) \\
\hline \multirow[t]{3}{*}{$\mathrm{Pd}_{80} \mathrm{Au}_{20}$} & $0.37 \pm 0.06$ & $4.00992(2)$ & 0.48 & 4.11 & $0.277(2)$ & $0.034(1)$ & 0.35 & 1.08 & 73 & 0.0772 & 4.66 & (a) \\
\hline & & & 0.48 & 4.11 & $0.246(1)$ & $0.036(1)$ & 0.32 & 1.00 & 67 & 0.0852 & 5.67 & (b) \\
\hline & & & $0.63(1)$ & $3.84(6)$ & $0.238(3)$ & $0.054(1)$ & 0.35 & 1.03 & 75 & 0.0766 & 4.59 & (c) \\
\hline \multirow[t]{3}{*}{$\mathrm{Pd}_{75} \mathrm{Au}_{25}$} & $0.38 \pm 0.02$ & $4.00620(2)$ & 0.49 & 4.26 & $0.170(1)$ & $0.082(1)$ & 0.33 & 1.02 & 73 & 0.0510 & 4.05 & (a) \\
\hline & & & 0.49 & 4.26 & $0.165(1)$ & $0.056(1)$ & 0.28 & 1.00 & 67 & 0.0538 & 4.5 & (b) \\
\hline & & & $0.57(1)$ & $4.24(9)$ & $0.188(4)$ & $0.069(2)$ & 0.33 & 1.24 & 75 & 0.0482 & 3.62 & (c) \\
\hline \multicolumn{13}{|c|}{ Loading pressure $=1.0 \mathrm{MPa}$} \\
\hline \multirow[t]{3}{*}{$\mathrm{Pd}_{90} \mathrm{Au}_{10}$} & $0.56 \pm 0.06$ & $4.02094(3)$ & 0.47 & 3.80 & $0.349(2)$ & $0.017(1)$ & 0.38 & 1.52 & 73 & 0.0368 & 2.39 & (a) \\
\hline & & & 0.47 & 3.80 & $0.305(1)$ & $0.002(1)$ & 0.31 & 1.00 & 67 & 0.0521 & 4.79 & (b) \\
\hline & & & $0.53(1)$ & $3.69(5)$ & $0.337(3)$ & $0.015(1)$ & 0.37 & 1.50 & 75 & 0.0367 & 2.37 & (c) \\
\hline \multirow[t]{3}{*}{$\mathrm{Pd}_{80} \mathrm{Au}_{20}$} & $0.41 \pm 0.06$ & $3.98198(3)$ & 0.48 & 4.11 & $0.192(2)$ & $0.028(2)$ & 0.25 & 1.12 & 73 & 0.1092 & 3.26 & (a) \\
\hline & & & 0.48 & 4.11 & $0.155(1)$ & $0.020(1)$ & 0.20 & 1.00 & 67 & 0.1183 & 3.82 & (b) \\
\hline & & & $0.64(1)$ & $3.79(13)$ & $0.157(4)$ & $0.031(2)$ & 0.22 & 1.02 & 75 & 0.1100 & 3.31 & (c) \\
\hline \multirow[t]{3}{*}{$\mathrm{Pd}_{75} \mathrm{Au}_{25}$} & $0.33 \pm 0.02$ & $4.00267(2)$ & 0.49 & 4.26 & $0.145(1)$ & $0.076(1)$ & 0.30 & 1.20 & 73 & 0.0584 & 5.98 & (a) \\
\hline & & & 0.49 & 4.26 & $0.137(2)$ & $0.038(1)$ & 0.21 & 1.00 & 67 & 0.0787 & 10.87 & (b) \\
\hline & & & $0.58(1)$ & $4.77(14)$ & $0.188(5)$ & $0.090(3)$ & 0.37 & 1.27 & 75 & 0.0567 & 5.64 & (c) \\
\hline
\end{tabular}

The three sets of parameters given for each sample correspond to (a) refinements using fixed average values for the temperature factors and including texture refinement, (b) refinements using fixed average values for the temperature factors without texture refinement, and (c) refinements including temperature factors and texture refinement; the numbers in parentheses are the estimated standard deviations in the last quoted digit, as given in GSAS for the refined parameters. 
when fixing or refining the $B_{\mathrm{M}}$ values. Therefore, the use of constant $B_{\mathrm{M}}$ values for all samples (without and with deuterium) is to be preferred in order to reduce the number of adjustable parameters, especially in view of the limited number of independent Bragg reflections in this high symmetry structure. However, the overall quality of the fits decreases dramatically when the texture corrections are not included (Table 1). The effect of including texture corrections is clearly visible in a reduction of the systematic overestimation of the $\{111\}$ peak intensities. Hence, texture corrections cannot be neglected. Comparing the different D-free alloys, because all samples are made of foil discs of the same thickness $(100 \mu \mathrm{m})$, the data in Table 1 indicate a decrease in the texture index with increasing Au content.

With the reliability obtained in the refinement procedure of the pure alloys in mind, the authors approached the analysis of the diffraction patterns of the more complex deuterated samples. The diffraction profiles of the deuterated samples indicate the presence of single-phase systems, which are modelled in the space group $F m \overline{3} m$ with deuterium atoms distributed to occupy the octahedral $(\mathrm{O})$ interstitial sites at $4 \mathrm{~b}$ Wyckoff positions and the tetrahedral (T) interstitial sites at $8 \mathrm{c}$ Wyckoff positions. The refined parameters for all the diffraction patterns of D-containing samples at $298 \mathrm{~K}$ are given in Table 2. In accordance with the interpretation of the D-free samples, three distinct refinements strategies were followed, i.e., $(a, b)$ refinements whereby the metal temperature factors $\left(B_{\mathrm{M}}\right)$ are fixed to the average values used for the D-free alloys (see previous paragraph), the deuterium temperature factors $\left(B_{\mathrm{O}}\right.$ and $\left.B_{\mathrm{T}}\right)$ are fixed to the averaged values obtained as described below, and the D-occupancy and all other parameters are refined with (a) or without (b) texture corrections, and (c) refinements whereby the metal and deuterium temperature factors are fitted together with D-occupancy, texture parameters and all other parameters. For all these cases, the metal content obtained from electron probe microanalysis is used without further refinement. The temperature factors for deuterium on the $\mathrm{O}$ and $\mathrm{T}$ interstitial sites were constrained to remain equal $\left(B_{\mathrm{O}}=B_{\mathrm{T}}\right)$. This assumption is made to stabilize the refinement and to reduce the correlations between the site occupancies and temperature factors. This is essential when one aims to assess the relative occupancy of the $\mathrm{O}$ and $\mathrm{T}$ sites by deuterium. This may be considered an oversimplification, as one could expect different vibrational amplitudes (temperature factors) for atoms in interstitials sites with different sizes. However, there is no information available to ensure physically reasonable values for $B_{\mathrm{O}}$ and $B_{\mathrm{T}}$, except perhaps a relationship based on the difference in size between $\mathrm{O}$ and $\mathrm{T}$ interstitial sites, as suggested in Ref. [22]. Moreover, refining $B_{\mathrm{O}}, B_{\mathrm{T}}$ and the site occupancies freely could lead to very different results. In general, a too high occupancy is set by spreading out the scattering power of a larger site, i.e., by a too large Debye-Waller factor. Therefore the assumption of equal temperature factors was preferred, and the refinements were done by alternatively fixing and varying the tempera- ture factors and site occupancies for the deuterium atoms until a smooth convergence is achieved for all diffraction patterns obtained in subsequent GEM runs. It was observed that the average values of temperature factors $\left(B_{\mathrm{O}}=B_{\mathrm{T}}\right)$ increase with increasing $\mathrm{Au}$ content. Further, they are nearly the same for samples with the same Au content loaded at different deuterium pressures. These averaged values of $B_{\mathrm{O}}=B_{\mathrm{T}}$ were fixed, while the site occupancies and other parameters were refined in the refinements (a) and (b) referred to above (i.e., refinements with and without texture corrections).

The data in Table 2 correspond to the best overall refinement of all data from various diffraction banks, and clearly indicate that a mixed deuterium occupancy of $\mathrm{O}$ and $\mathrm{T}$ interstitial sites provides the best description for all samples. Structural models including only O-site occupancy were also considered, but they were found to give a poorer description of the experimental data, as shown in Fig. 4 and discussed in a following paragraph. The data in Table 2 indicate that the fit quality does not differ significantly when fixing or refining the thermal parameters. Small differences in D-occupancies can be seen in Table 2. Similar refinement quality but slightly lower occupancies (see Fig. 5) were also obtained by setting $B_{\mathrm{T}}=B_{\mathrm{O}} / 3.41$, as suggested in Ref. [22] based on the difference in size between $T$ and $\mathrm{O}$ interstitial sites in pure $\mathrm{Pd}$. The addition of the texture corrections, however, seems to be essential for a proper description of the diffraction patterns. Still, refinements without texture corrections lead to the same conclusions regarding the deuterium site occupancies and their correlation with the Au content (see Table 2 and Fig. 5).

The deuterium content, as calculated from the total sum of the site occupancies of all $\mathrm{O}$ and $\mathrm{T}$ sites, decreases steadily with increasing $\mathrm{Au}$ content. This is in good agreement

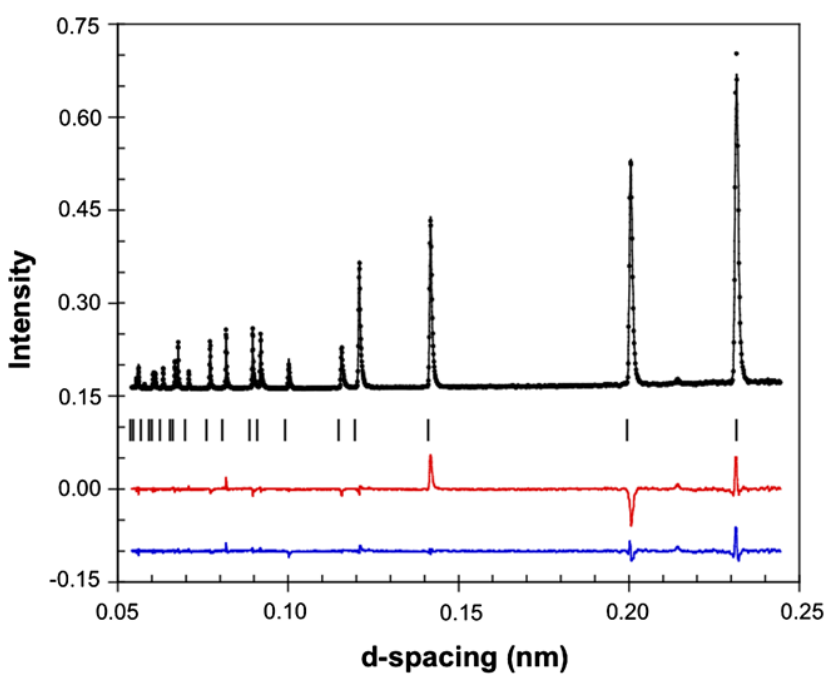

Fig. 4. ND pattern of $\mathrm{Pd}_{75} \mathrm{Au}_{25}$ charged with $\mathrm{D}_{2}$ at $0.6 \mathrm{MPa}$. The upper symbols and line represent the observed and calculated patterns $(\mathrm{O}+\mathrm{T}$ case). The two difference profiles $\left(I_{\mathrm{obs}}-I_{\text {calc }}\right)$ plotted below relate to the case where deuterium occupies the $\mathrm{O}$ sites only (upper curve) and the $\mathrm{O}+\mathrm{T}$ sites (lower curve), respectively. 


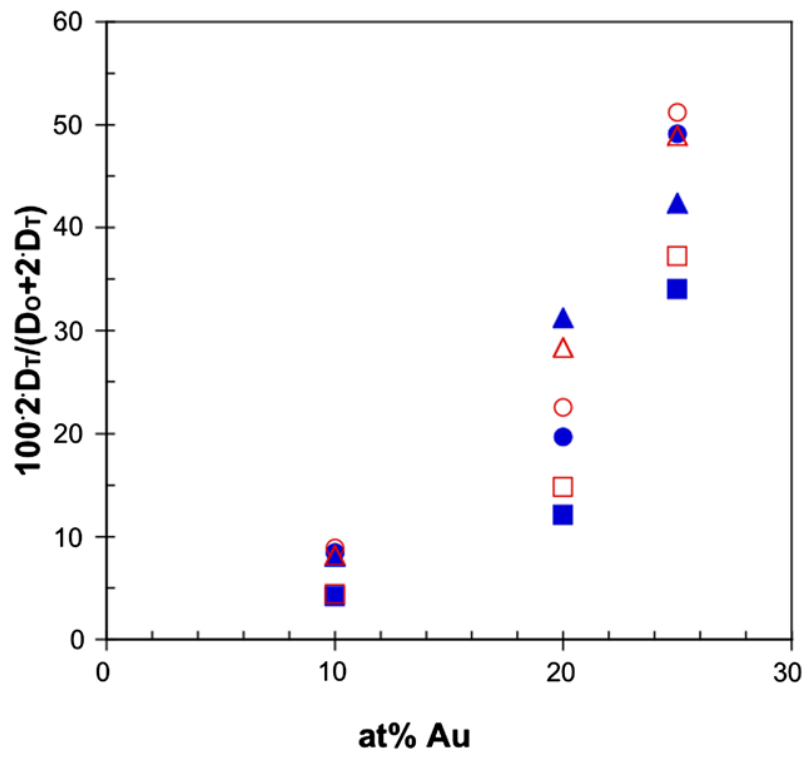

Fig. 5. Deuterium T-site occupancy relative to total deuterium content as obtained from refinements using fixed average values for the temperature factors and including texture refinement (circles), from refinements including temperature factors and texture refinement (triangles), and from refinements considering $B_{\mathrm{T}}=B_{\mathrm{O}} / 3.41$ (squares). The compositions of samples loaded at 0.6 and $1.0 \mathrm{MPa}$ are represented by solid and open symbols, respectively.

with the trend determined from the TDS analysis (Fig. 2). The deuterium content estimated from the Rietveld profile analysis is slightly lower than the deuterium content estimated by thermal desorption measurements. The lower values are not surprising, because the ND data probes the $\mathrm{D}$ content at the $\mathrm{O}$ and $\mathrm{T}$ lattice positions, while the TDS technique determines the overall $\mathrm{D}$ content, including also the deuterium located at the surface, subsurface, grain boundaries, dislocations and defects.

Furthermore, it is important to note that the occupancy of $\mathrm{O}$ sites gradually decreases, while the occupancy of $\mathrm{T}$ sites continuously increases with increasing $\mathrm{Au}$ content. In the case of $\mathrm{Pd}_{90} \mathrm{Au}_{10}-\mathrm{D}$ alloys, only $\sim 8 \%$ of $\mathrm{D}$ can be found on the $\mathrm{T}$ site. However, this increases to a significantly higher fraction $(\sim 50 \%)$ for $\mathrm{Pd}_{75} \mathrm{Au}_{25}-\mathrm{D}$ alloys (Fig. 5). Without including T-site occupancy, it is impossible to achieve a satisfactory fit of the diffraction data, especially for the samples with a high Au content. As an illustrative example of the results of Rietveld profile analysis, Fig. 4 shows a representative diffraction pattern and analysis for a $\mathrm{Pd}_{75} \mathrm{Au}_{25}$ sample charged with $\mathrm{D}_{2}$ at $0.6 \mathrm{MPa}$. The two difference profiles plotted below the observed and calculated patterns correspond to the cases where deuterium occupies the $\mathrm{O}$ sites only (upper curve) and $\mathrm{O}+\mathrm{T}$ sites (lower curve). The same texture parameters are used to calculate these profiles, hence the differences are only due to neglecting or including T-site occupancy in the analysis. When only the O-site occupancy is considered, major differences between the calculated and observed intensities for the $\{200\}$ and $\{220\}$ reflections are evident. Assuming that deuterium also occupies the T-interstitial sites significantly reduces these differences and improves the overall quality of the fit significantly. Structure factor calculations indeed show that the $\mathrm{T}$ occupancy decreases the intensity of the $\{200\}$ reflections and increases the intensity of the $\{220\}$ reflections, while leaving the $\{111\}$ reflections unchanged.

The data presented in Table 2 and Figs. 4 and 5 support the conclusion that all the samples investigated here were single-phase systems, which can only be adequately described by considering a simultaneous occupation of $\mathrm{O}$ and $\mathrm{T}$ sites. This is the case not only for samples loaded in supercritical conditions $\left(\mathrm{Pd}_{75} \mathrm{Au}_{25}\right)$, but also for the samples loaded in subcritical conditions, i.e., in the $\beta$-hydride phases $\left(\mathrm{Pd}_{90} \mathrm{Au}_{10}\right.$ and $\left.\mathrm{Pd}_{80} \mathrm{Au}_{20}\right)$. Clearly, a simultaneous occupancy of $\mathrm{T}$ positions in addition to $\mathrm{O}$ interstices is therefore characteristic not only of samples prepared under supercritical conditions, as inferred in Ref. [22] for Pd-D. In the case of $\mathrm{Pd}-\mathrm{Au}$ alloys, the $\mathrm{T}$-site occupancy is probably closely related to the local atomic composition around the sites, which makes them more favourable for $H(D)$ occupation. First principles calculations for hydrogen in $\mathrm{Pd}-\mathrm{Au}$ and $\mathrm{Pd}-\mathrm{Ag}$ alloys support this statement $[17,18]$, showing that the $\mathrm{O}$ site is preferred when most of the neighbours are palladium atoms, while the $\mathrm{T}$ site is preferred when the alloying element dominates. The increase in the T-site occupancy determined from the present ND study suggests that the occupation of $\mathrm{T}$ sites become energetically more favourable for D absorption with increasing Au content. This is in good agreement with recent results from first principles calculations [17], which indicate that, even at low $\mathrm{H}$ content, $\mathrm{H}$ absorption at the $\mathrm{T}$ sites can become more favourable than at the $\mathrm{O}$ sites, depending on the type and concentration of alloying element. Though the results from these first principles calculations concern $\mathrm{H}$ and not $\mathrm{D}$, it is likely that the T-site occupancy will be even more favourable for the heavier isotope because of its lower vibrational energy. Further analysis of the ND data is required to obtain information on the local atomic configuration, but this cannot be obtained from the present full-profile analysis. This issue deserves further studies, and will be discussed in a future paper.

\section{Conclusions}

This study provides information about the phases formed during deuterium absorption/desorption in $\mathrm{Pd}-$ Au-alloys, the deuterium site occupancy and its relation with the desorption characteristics.

First, it is shown that the hydrogen (deuterium) desorption characteristics change with the content of gold. Desorption from $\mathrm{Pd}-\mathrm{Au}$ alloys deuterated initially in the single $\beta$-hydride phase occurs via a two-stage process, i.e., (i) desorption from interstitial sites of the single-phase $\beta$ hydride and (ii) desorption from a system comprising coexisting $\alpha$ and $\beta$ domains with deuterium release accompanied by a $\beta \rightarrow \alpha$ phase transformation reaction. Desorption from alloys in a single-supercritical phase is a single-stage 
process i.e., desorption from interstitial sites of the supercritical solid solution phase, which is quite similar to desorption from the single $\beta$-hydride phase.

Second, the analysis of the measured ND data confirms that the same types of interstitial sites are occupied in both the (subcritical) $\beta$-hydride phase and in the (supercritical) solid solution phase. Only Rietveld refinement including mixed occupation of $\mathrm{O}$ and $\mathrm{T}$ sites could describe the ND data adequately. With increasing $\mathrm{Au}$ content, the occupancy of the $\mathrm{O}$ sites decreases and that of the $\mathrm{T}$ sites increases.

\section{Acknowledgements}

The authors gratefully acknowledge Ing. C. Kwakernaak from the Department of Material Science and Engineering of Delft University of Technology for electron probe microanalysis. This work is part of a joint research programme of the Materials innovation institute (M2i) (the former Netherlands Institute for Metals Research), and the Stichting voor Fundamenteel Onderzoek der Materie (FOM) financially supported by the Nederlandse Organisatie voor Wetenschappelijk Onderzoek (NWO). Further financial support was received through SenterNovem. The neutron diffraction work at ISIS was made possible through a NWO Grant.

\section{References}

[1] Paglieri SN, Way JD. Sep Purif Methods 2002;31:1.

[2] Sholl DS, Ma YH. MRS Bull 2006;31:770.

[3] Lee S-M, Flanagan TB, Kim G-H. Scripta Met et Mat 1995;32:827.

[4] Massalski TB, Okamoto H, editors. Binary alloy phase diagrams, 2nd ed., Vol. 1-3. Metals Park (OH): ASM International; 1990.

[5] Worsham Jr JE, Wilkinson MK, Shull CG. J Phys Chem Solids 1957;3:303.

[6] Ferguson GA, Schindler AJ, Tanaka T, Morita T. Phys Rev A 1965; $137: 483$

[7] Nelin G. Phys Stat Sol (b) 1971;45:527.
[8] Wu E, Kennedy SJ, MacGray EA, Kisi EH. J Phys: Condens Matter 1996;8:2807.

[9] Bergsma J, Goedkoop JA. Physica 1960;26:744.

[10] Rowe JM, Rush JJ, de Graaf LA, Ferguson GA. Phys Rev Lett $1972 ; 29: 1250$

[11] Sköld K. Quasielastic neutron scattering studies of metal hydrides. In: Alefeld IG, Völkl J, editors. Hydrogen in metals. Berlin: Springer; 1978. p. 267.

[12] Sköld K, Nelin G. J Phys Chem Solids 1967;28:2369.

[13] Maeland A. Can J Phys 1967;46:121.

[14] Elsässer C, Ho KM, Chan CT, Fähnle M. Phys Rev B 1991;44:10337.

[15] Krimmel H, Schimmele L, Elsässer C, Fahne M. J Phys: Condens Matter 1994;6:7679.

[16] Gillan MJ. J Phys C: Solid State Phys 1986;19:6169.

[17] Sonwane CG, Wilcox J, Ma YH. J Chem Phys 2006;125:184714; Sonwane CG, Wilcox J, Ma YH. J Phys Chem B 2006;110: 24549.

[18] Lovvik OM, Olsen RA. J Alloys Compd 2002;330-332:332.

[19] Kamakoti P, Sholl DS. J Membr Sci 2003;225:145; Kamakoti P, Sholl DS. Phys Rev B 2005;71:014301.

[20] Ke X, Kramer GJ. Phys Rev B 2002;66:184304.

[21] Beg MM, Ross DK. J Phys C 1970;3:2487.

[22] Pitt MP, MacGray EA. Europhys Lett 2003;64:344.

[23] Singh S, Eijt SWH, Zandbergen MW, Legerstee WJ, Svetchnikov VL. J Alloys Compd 2007;441:344.

[24] Williams WG, Ibberson RM, Day P, Enderby JE. Physica B 1998;241-243:234.

[25] Larson AC, Dreele RBV, General structure analysis system (GSAS), LAUR 86-748, Los Alamos National Laboratory, 1994.

[26] Sakamoto Y, Chen FL, Ura M, Flanagan TB. Ber Bunsenges Phys Chem 1995;99:807.

[27] Lukaszewski M, Kusmierczyk K, Kotowski J, Siwek H, Czerwinski A. J Solid State Electrochem 2003;7:69.

[28] Turnbull A, Hutchings RB, Ferriss DH. Mat Sci Eng A 1997;238:317.

[29] Stern A, Resnik A, Shaltiel D. J Phys F: Met Phys 1984; $14: 1625$.

[30] Sakamoto Y, Yuwasa K, Hirayama K. J Less-Common Met 1982;88:115.

[31] Maeland A, Flanagan TB. J Phys Chem 1965;69:3575.

[32] Peng L-M, Ren G, Dudarev SL, Whelan MJ. Acta Cryst A 1996;52:456.

[33] Baranowski B, Majchrzak S, Flanagan TB. J Phys F 1971;1:258. 